\title{
COVID-19 vaccines: ethical framework concerning human challenge studies
}

\author{
Daniela Calina ${ }^{1}$ (D) - Thomas Hartung ${ }^{2,3}$ - Anca Oana Docea ${ }^{4}$ - Demetrios A. Spandidos ${ }^{5}$ - Alex M. Egorov ${ }^{6,7}$. \\ Michael I. Shtilman ${ }^{8} \cdot$ Felix Carvalho $^{9} \cdot$ Aristidis Tsatsakis $^{7,10,11,12}$
}

Received: 8 July 2020 / Accepted: 21 August 2020 / Published online: 27 August 2020

(C) Springer Nature Switzerland AG 2020

\begin{abstract}
Background The pandemic associated with the new SARS-CoV-2 coronavirus continues to spread worldwide. The most favorable epidemic control scenario, which provides long-term protection against COVID-19 outbreak, is the development and distribution of an effective and safe vaccine. The need to develop a new COVID-19 vaccine is pressing; however, it is likely to take a long time, possibly several years. This is due to the time required to demonstrate the safety and efficacy of the proposed vaccine. and the time required to manufacture and distribute millions of doses.

Objectives To accelerate this development and associated safety testing, the deliberate infection of healthy volunteers has been suggested. The purpose of this short communication is to describe the ethical aspects of this type of testing,

Results Deliberate infection of volunteers with a dangerous virus such as SARS-CoV-2 was initially considered unethical by researchers; but the current pandemic is so different from previous ones that these studies are considered ethical if certain criteria are met. Participants in human challenge studies must be relatively young, in good health and must receive the highest quality medical care, with frequent monitoring. Tests should also be performed with great caution and specialized medical supervision. Besides, the fact that obtaining vaccines faster through deliberate infection studies of healthy people has greater benefits than risks, has been demonstrated by obtaining other vaccines in other historical pandemics such as: smallpox, influenza, malaria, typhoid fever, Dengue fever and Zika.

Conclusions One possibility to shorten the time required for the development of COVID-19 vaccines is to reduce clinical phases II and III by using human challenge studies through eliberate infection of healthy volunteers with SARS-CoV-2 after administration of the candidate vaccine. Accelerating the development of a COVID-19 vaccine even for a few weeks or months would have a great beneficial impact on public health by saving many lives.
\end{abstract}

Daniela Calina, Thomas Hartung and Anca Oana Docea contributed equally to this work.

Daniela Calina calinadaniela@gmail.com

$\triangle$ Aristidis Tsatsakis tsatsaka@uoc.gr

1 Department of Clinical Pharmacy, University of Medicine and Pharmacy of Craiova, 200349 Craiova, Romania

2 CAAT-Europe at the University of Konstanz, 78457 Constance, Germany

3 CAAT, Bloomberg School of Public Health, Johns Hopkins University, Baltimore, MD 21287, USA

4 Department of Toxicology, University of Medicine and Pharmacy of Craiova, 200349 Craiova, Romania

5 Laboratory of Clinical Virology, Medical School, University of Crete, 71409 Heraklion, Greece
6 FSBSI "Chumakov Federal Scientific Center for Research and Development of Immune- and Biological Products of Russian Academy of Sciences", 108819 Moscow, Russia

7 Russian Academy of Sciences, Moscow, Russia

8 D.I. Mendeleyev University of Chemical Technology, 125047 Moscow, Russia

9 UCIBIO, REQUIMTE, Laboratory of Toxicology, Department of Biological Sciences, Faculty of Pharmacy, University of Porto, 4050-313 Porto, Portugal

10 Department of Analytical and Forensic Medical Toxicology, Sechenov University, 119991 Moscow, Russia

11 Department of Forensic Sciences and Toxicology, Faculty of Medicine, University of Crete, 71003 Heraklion, Greece

12 Laboratory of Toxicology, Medical School, University of Crete, 70013 Heraklion, Greece 
Keywords COVID-19 vaccines $\cdot$ Risk taking $\cdot$ Human challenge studies $\cdot$ Randomized clinical trials

\section{Introduction}

For COVID-19 disease, caused by the SARS-CoV-2 coronavirus, there is still no approved curative treatment available to fight the infection and no vaccine to immunize the population against the virus [1]. Medical practitioners worldwide have repurposed various existing drugs for the treatment of patients with COVID-19, with various effects and difficulties in the assessment of the treatment efficacy [2]. As the COVID-19 pandemic spreads worldwide, acceleration in obtaining an ideal vaccine against SARS-CoV-2 has become the critical demand of medical practitioners and scientists, even if this might involve a more 'relaxed' approach to established procedures [3]. Notably, already in early April 2020, 105 vaccine development projects were counted [4].

For the discovery of a new COVID-19 vaccine, the process from the fundamental concept to the administration of the vaccine preparation to the population is a long and tedious one [5]. The purpose of vaccination is to ensure effective prevention [6]. This journey can take a long time, even decades, as the logical reasoning on the mechanisms of action of the newly designed molecule, investigates the toxicity and immunogenicity characteristics of the preparation [7]. It takes typically, on average 10.7 years [8]. New drugs/molecules usually are initially tested on animal models, through preclinical studies, and at a later stage in humans, through clinical studies/trials [9]. Intending to expedite the process of obtaining COVID-19 vaccines, a unique situation has arisen: animal testing is not being performed [10]. This is in part because animal models of COVID-19 are only emerging. The problem is to find an animal that reflects not only significant aspects of COVID-19 pathology but also a similar immune response to the vaccine candidate. This opens up for the use of new approach methods, i.e., alternatives to animal experiment [11].

Clinical studies involving vaccines have the same methodology as other drug development processes: In phase I, the safety profile of the vaccine is examined on healthy volunteers. Subsequently, in phase II, the adverse reactions are further investigated, together with the immune system reaction and the identification of the appropriate dose and frequency of administration. Last but not least, in phase III, the effectiveness of the vaccine is verified to determine its effectiveness in preventing infection with the targeted virus [11]. A major difference though, is the preventive application of a vaccine [12]. This means that typically a large population of healthy people, often a group of high risk patients of the infection to be studied, are vaccinated to quantify the effect of vaccination on the probability of contracting the infection and the severity of its course [13]. Obtaining a vaccine against the novel coronavirus is a time-consuming issue, mainly due to the long duration of tests performed in the third stage of obtaining a vaccine. It is that stage in which thousands or tens of thousands of individuals are voluntarily being included in the testing of the product [14].

In this process, some receive the experimental vaccine, while others do not; the results involve determining whether those who received it become immune to coronavirus. However, this is a time-consuming process, and scientists, under the pressure of the pandemic, are considering whether this test should be replaced with another. The variant would be human testing on a small group of volunteers by administering the vaccine following exposure to the coronavirus, to determine whether they become immune to the virus [15].

The current prevalence of COVID-19 in the US population, for example, is about 40 in 10,000 people as per 30 May 2020 according to the US Center for Disease Control (CDC) [16]. This means that 10,000 people have to be studied to observe less than 40 infections, which usually would occur. With the current low numbers in new infections in Europe, such studies can hardly be done: infections peaked on 9 April, and declined by $80 \% 2$ months later [15] with a prevalence until now of 2.8 per 10,000. Using high risk groups such as health care workers improve somewhat the statistical odds, but these simple number plays may serve to illustrate the challenge.

Taking further into account that no vaccine is $100 \%$ effective, it becomes even more challenging: If we take influenza as an example, over the last decade, CDC reported efficacy for influenza vaccine between 19 and 60\% [16]. This represents an enormous statistical challenge, given besides substantial limitations of COVID-19 test accuracies.

In the search for the ideal COVID-19 vaccine, some researchers have proposed expediting the standard procedure for validating a vaccine proposal by voluntarily exposing healthy individuals to COVID-19 [17]. Deliberate infection of individuals is a challenge in the context in which there is no approved drug or antibody therapy for COVID-19 disease.

Authorities in the medical field who support the benefits of SARS-CoV-2 infection studies describe a crucial advantage: accelerating the process of studying and approving an effective vaccine. However, the question of whether voluntary infection with COVID-19 is ethical needs to be addressed [18]. Assuming again, the efficacy of the influenza vaccine would imply a substantial number of volunteers going through a full infection and perhaps at even higher rate considering that these are experimental vaccines only. Given the case of influenza vaccination fatality rate of about $0.2 \%$ in the age groups from 20 to 30 [19] the most likely age groups to be considered for such courageous experiments, this means that likely 2 out 
of 1000 volunteers would die without vaccine efficacy and one assuming efficacy [20].

Our most recent research [21] indicates that SARS-CoV-2 infects human brain cells, in line with a high prevalence of neurological symptoms in patients. As the human brain is immune privileged, i.e., immune cell invasion and inflammation are rarely observed, it is not clear whether such infection is eradicated. This illustrates that the questions for these volunteers is not only to take a risk and die or survive, but they could also suffer from chronic infection with possible impact on long-term health such as the progression of neurodegenerative diseases [21].

On the positive side, in addition to vaccines, infection studies can prove to be helpful for the following reasons: i) to better understand the immune process developed in response to the infection; ii) validate diagnostic tests; iii) provide more data on the dynamics of the infectious process; iv) determine whether the individual can transmit the virus following the remission of symptoms [22].

Human challenge studies have been used successfully in history for smallpox, yellow fever, and malaria. More recent examples of infectious diseases whose vaccines have been developed more rapidly using this methodology include typhoid fever, cholera, and influenza [23].

\section{Ethical recommendations for planning a possible SARS-CoV-2 infection study}

It is challenging to develop an infection study methodology that respects the principles of modern medical ethics, and at a superficial glance, it may seem almost impossible. However, experts from the World Health Organization and other institutions have developed a series of principles that, once followed, ensure the safe conduct of the study, without limiting the rights and freedoms of the volunteers included. A study involving the intentional exposure of volunteers to the virus requires sound scientific justification, and it must have the potential to make a significant contribution to a critical public health issue [24].

In other words, it must bring significant amount of new data and make a decisive contribution to the development and use of new vaccines or public health policies - data that could not be obtained by other methods without affecting speed or efficiency [24].

Scientific justification A study involving the intentional exposure of volunteers to the virus requires strong scientific justification, must bring a significant amount of new data and make a decisive contribution to the development and use of new vaccines or public health policies - data that could not be obtained by other methods, without affecting speed or efficiency [24].
Risk and benefit balance The benefits of such a study must outweigh the risks, not only for the participants but also for society and the community. The efforts of researchers must continuously focus on minimizing the risks and maximizing the benefits, comparing this balance with that of other study methodologies. It is recommended to quantify these abstract notions accurately, for example, the benefit consists in an exact number of people whose infection and severe evolution was prevented by the development of that vaccine, an appearance on the market of the vaccine in a shorter period, and a more rapid social and economic recovery [25].

The risks for volunteers with the expected benefit to society of discovering a treatment are weighed. These 'human challenges' must be ruled out in the case of diseases, such as anthrax or Ebola, with a high mortality rate [25].

The study methodology should be discussed, and representatives of society, medical practices, regulators and the pharmaceutical industry should work together to adapt this. All these stakeholders have the role of ensuring the acceptability in the community, the transparency and the minimal impact of the research, through the most rapid method, at a local and international level. To ensure the maximum beneficial impact of infection studies, they must be integrated into a coherent research program. They must be carried out in such a manner as to affect society as a whole, and the results obtained should be published as soon as possible. Subsequently, good cooperation with regulatory bodies is warranted (which must analyze the data and reach a decision on the approval of vaccines) [25].

Choosing the most appropriate organizer of the study To ensure the scientific rigor and safety of the volunteers, the study must be conducted in a carefully selected institution. This should include laboratories capable of producing and safely administering the vaccine and the targeted coronaviral strain, as well as performing all necessary tests at the state-ofthe-art quality (PCR diagnosis, serological tests, general assessment of the biological status of the volunteers). Volunteer isolation and prompt treatment facilities may also be required, including intensive care units [26].

Collaboration of researchers In order to ensure the maximum beneficial impact of infection studies, they must be integrated into a coherent research program, conducted in such a way as to affect the normal functioning of society in the least possible way, and to publish the results obtained as soon as possible. Subsequently, good collaboration with regulatory bodies is needed (which must analyze the data and make a decision on the approval of vaccines) [26].

The right candidate for inclusion in study From the epidemiological data on SARS-CoV-2 obtained to date, it can be deduced that a young adult, without comorbidities, has 
minimal risk in the short- and long-term, if he suffers from the infection [27].

The challenges that medical specialists will face are not limited to ethics, but also to the criteria for accepting volunteers into the program. Volunteers must be between the ages of 18 and 30 and must be clinically healthy without adjacent conditions. Also, ethical principles dictate that this volunteer is already at medium-high risk of infection (COVID-19 highrisk residents), for the additional risk posed by the study to be minimal. In addition to the ideal candidate, the ideal institution that would organize the study is also important: It must allow the continuous, careful monitoring of volunteers, and have the capacity for supportive treatment established rapidly, including intensive care if necessary [27].

Study participants To ensure the minimal risk to which volunteers can be subjected, it is vital that they are selected according to rigorous criteria. First, the recommended age group should be between 18 and 30 years [28]. Subsequently, priority should be given to volunteers who are already at a high risk of being infected in the community which leads to a minimal additional risk caused by the study. Second, volunteers may be selected from people who have already been exposed to the new coronavirus infection. Volunteers should come from areas profoundly affected by the coronavirus epidemic in order to ensure the natural likelihood of the infection. Through extensive medical tests, it can be guaranteed that none of the participants have comorbidities or conditions that are known to increase the risks of COVID-19, such as respiratory issues. These volunteers should also be prioritized to obtain the best possible treatment for complications following coronavirus exposure. Following the completion of the experiment, participants should remain isolated from the remainder of the population for 14 days, which corresponds to the period when they could be contagious, to minimize the risk of the contamination of relatives [28].

Exclusion criteria must take into account, including the risk factors identified by epidemiological studies, and must be adapted as new data emerge.

Informed consent Volunteers must accurately understand the risks associated with such a study and participate only if they have assessed the risk-benefit balance, but also the unknowns associated with a new virus such as SARSCoV-2. The consent must also be provided during the study, as new data emerge. Researchers considering the study of infection also suggest a preliminary step, to expose a separate group of volunteers to the virus, to determine the amount of virus required for infection, and to confirm that no more severe forms of the disease develop through infection. This stage follows an ethical principle of minimizing the risk associated with participation [29].
A special attention must be given to volunteers from vulnerable populations with resource-poor settings. The ability to provide informed consent rests on two pillars: the ability to properly understand the potential risks and benefits related to the proposed trial, and the ability to refuse participation. On both accounts, volunteers in resource-poor settings are vulnerable: their literacy levels may not enable them to properly understand what risks they may expose themselves to, and the financial compensation provided for their participation can become an undue financial inducement if they are in no position to refuse it [29]. An added complication is that some participants covertly enrol in several studies simultaneously, in order to increase their income, conceal underlying medical conditions, intake of concomitant medications, or substance abuse. This may expose the volunteers to medical risks, and potentially biases study data $[28,29]$.

Data analysis The methodology and results of the study should be reviewed by an independent committee of experts, such as epidemiologists, infectious disease experts, clinical pharmacists, virologists, bioethics experts, etc. (Fig. 1).

\section{Discussion}

Historically speaking, the 'human challenge studies' approaches are not at all unprecedented. Vaccine studies have only recently been standardized and apply extremely demanding ethical requirements to minimize the risks. In the early 1970s, a French hepatitis B vaccine was first tested on volunteers from the medical staff most exposed to the virus [30].

The path of a vaccine from laboratory testing to the population in need begins to become complex only when its effects on humans begin to be studied. Testing with human subjects has long been a part of the history of medicine. The deliberate infection of volunteers provides more rapid results, although inevitably, it also involves certain risks [27].

The human challenge is the contamination of 100 healthy volunteers, some of whom would receive a candidate vaccine, i.e., at an advanced stage of testing, in order to expedite the process of establishing a treatment, which will become available to the general public [24].

New approach methods might accelerate preclinical testing [6] this approach renders unnecessary the first stages of testing of the usual procedure - in the laboratory, then on animals. However, it is very different when it comes to testing on humans, which is the most time-consuming stage. Traditionally, the candidate vaccine is widely administered to a population of approximately 3000 individuals [11]. These individuals then return to their healthy lives, and researchers then aim to determine whether those receiving the treatment are more resistant to the disease than those receiving the placebo. The main difference from the approach promoted 
Fig. 1 Key points of a possible SARS-CoV-2 infection study

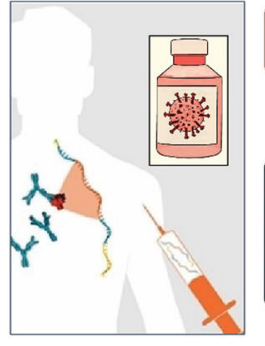

Ethical principles underlying a SARS-CoV-2 infection study

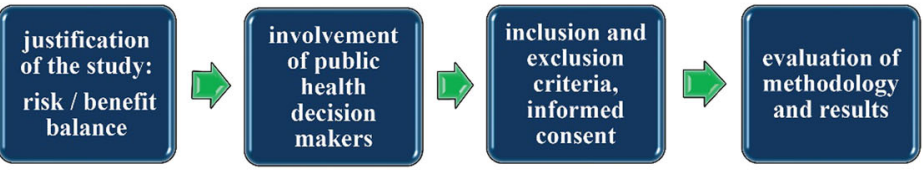

by researchers is that it is not the medical practitioners who expose the volunteers directly to the disease.

\section{Conclusions and future perspectives}

Increasingly, policy-makers, scientists and general public are claiming that the world is in dire need of an COVID-19 vaccine, warranting the risk of volunteer infection studies; thus, regulators and funders of research projects should already begin the necessary preparations, weighing whether such studies can commence with human infection after potential vaccines have completed the initial safety tests.

The use of tests with human volunteers would be useful in order to expedite the procedures for the development of a COVID-19 vaccine if such a project is approved by ethical specialists. On the other hand, the deliberate infection of individuals poses a problematic ethical issue, in the context in which there is no drug or functional therapy for the treatment of SARS-CoV-2 infection.

Despite these benefits and the tradition that would militate in favor of taking these risks to the health and lives of volunteers, this type of experimental approach faces severe ethical obstacles. Deliberate infection of individuals is a challenge in the context in which there is no approved drug or antibody therapy for COVID-19 disease. In case of infection studies, after the administration of the vaccine, the volunteer is exposed in a controlled way to the virus targeted by the vaccine. Thus, conclusions can be drawn from the study of a smaller number of volunteers, and the vaccine that offers the most sustained protection will differentiate more clearly and faster from other vaccines studied. An infection study can play the role of phase III testing, or it can act as an intermediate stage, accelerating phase III, which selects the most viable candidates from the developed vaccines.

However, such studies must offer both volunteers and professionals involved maximum protection against any effect of infection, including the long-term health outcomes, which are only becoming known. Although this procedure raises serious ethical concerns, medical authorities who support the benefits of voluntary SARS-CoV-2 infection studies describe a crucial advantage: speeding up the study process of approving an effective vaccine.

\section{References}

1. Docea AO, Tsatsakis A, Albulescu D, Cristea O, Zlatian O, Vinceti $\mathrm{M}$, et al. A new threat from an old enemy: re-emergence of coronavirus (review). Int J Mol Med. 2020;45:1631-43.

2. Nitulescu G, Paunescu H, Moschos S, Petrakis D, Nitulescu G, Ion $\mathrm{GN}$, et al. Comprehensive analysis of drugs to treat SARS-CoV-2 infection: mechanistic insights into current COVID-19 therapies (review). Int J Mol Med. 2020;46:467-88.

3. Tsatsakis A, Petrakis D, Nikolouzakis TK, Docea AO, Calina D, Vinceti M, et al. COVID-19, an opportunity to reevaluate the correlation between long-term effects of anthropogenic pollutants on viral epidemic/pandemic events and prevalence. Food Chem Toxicol. 2020;141:111418.

4. Arsene AL, Dumitrescu IB, Dragoi CM, Udeanu DI, Lupuliasa D, Jinga $\mathrm{V}$, et al. A new era for the therapeutic management of the ongoing COVID-19 pandemic. Farmacia. 2020;68:185-96.

5. Offit PA. Why are pharmaceutical companies gradually abandoning vaccines? Congress has the power to protect vaccines, a product that is vital to the health of the United States and the rest of the world. Health Aff. 2005;24:622-30.

6. Busquet F, Hartung T, Pallocca G, Rovida C, Leist M. Harnessing the power of novel animal-free test methods for the development of COVID-19 drugs and vaccines. Arch Toxicol. 2020;94:2263-72.

7. Jamrozik E, Selgelid MJ. COVID-19 human challenge studies: ethical issues. Lancet Infect Dis. 2020;20(8):e198-203.

8. Torequl Islam M, Nasiruddin M, Khan IN, Mishra SK, Kudrat-EZahan M, Alam Riaz T, et al. A Perspective on Emerging Therapeutic Interventions for COVID-19. Front Public Health. 2020;3(8):281.

9. Docea AO, Gofita E, Calina D, Zaharie SI, Valcea DI, Mitrut P. Autoimmune disorders due to double antiviral therapy with Peginterferon and ribavirin in patients with hepatitis $\mathrm{C}$ virus infection. Farmacia. 2020;64:605-11.

10. Lakdawala SS, Menachery VD. The search for a COVID-19 animal model. Science. 2020;368:942-3.

11. Calina D, Docea AO, Petrakis D, Egorov AM, Ishmukhametov AA, Gabibov AG, et al. Towards effective COVID-19 vaccines: updates, perspectives and challenges (review). Int J Mol Med. 2020;46:3-16.

12. Rostami-Nejad M, Yazdi MH, Nikfar S, Rezaie A, Abdollahi M. Potential vaccines for treating Crohn's disease. Iran Biomed J. 2020;24:1-14.

13. Stokes EK, Zambrano LD, Anderson KN, Marder EP, Raz KM, Suad El Burai F, et al. Coronavirus Disease 2019 Case Surveillance 
— United States, January 22-May 30, 2020. MMWR Morb Mortal Wkly Rep. 2020;69:759-65.

14. Rapid Risk Assessment: Coronavirus disease 2019 (COVID-19) in the EU/EEA and the UK - tenth update. https://www.ecdc.europa. $\mathrm{eu} / \mathrm{en} /$ publications-data/rapid-risk-assessment-coronavirus-disease2019-covid-19-pandemic-tenth-update. Accessed 19 June 2020.

15. World Health Organization: Draft landscape of COVID-19 candidate vaccines - 15 May 2020. Who, 2020. https://www. chathamhouse.org/expert/comment/hurdles-developing-covid-19vaccine-why-international-cooperation-needed?gclid= E A I a I Q o b C h M I 4 d K a oys6gIVCRd7Ch3ydQv0EAAYASAAEgLi9vD_BwE. Accessed 11 June 2020.

16. CDC Seasonal Flu Vaccine Effectiveness Studies | CDC. https:// www.cdc.gov/flu/vaccines-work/effectiveness-studies.htm. Accessed 15 May 2020.

17. Movert E, Wu Y, Lambeau G, Kahn F, Touqui L, Areschoug T. Using patient pathways to accelerate the drive to ending tuberculosis. J Infect Dis. 2013;208:2025-35.

18. Pronker ES, Weenen TC, Commandeur H, Claassen EH, Osterhaus AD. Risk in vaccine research and development quantified. PLoS One. 2013;8(3):e57755.

19. Mortality Risk of COVID-19 - Statistics and Research - Our World in Data. https://covid19.who.int/?gclid=EAIaIQobChMI1brnrY2 s6gIVxgJ7Ch2iFwLyEAAYASAAEgILofD_BwE. Accessed 12 June 2020.

20. Plotkin SA, Caplan A. Extraordinary diseases require extraordinary solutions. Vaccine. 2020;38(24):3987-8.

21. Bullen CK , Hogberg HT , Bahadirli-Talbott A, Bishai WR, Hartung T, Keuthan C. Infectability of Human BrainSphere Neurons Suggests Neurotropism of SARS-CoV-2. ALTEX -
Alternatives to animal experimentation. 2020; https://doi.org/10. 14573/altex.2006111.

22. Delany I, Rappuoli R, De Gregorio E. Vaccines for the 21 st century. EMBO Mol Med. 2014;6:708-20.

23. Bompart F. Healthy volunteers for clinical trials in resource-poor settings: National registries can address ethical and safety concerns. In: Cambridge Quarterly of Healthcare Ethics. vol. 28.2019; Cambridge University Press, pp134-143.

24. Bambery B, Selgelid M, Weijer C, Savulescu J, Pollard AJ. Ethical criteria for human challenge studies in infectious diseases. Public Health Ethics. 2016;9(1):92-103.

25. Eyal N, Lipsitch M, Smith PG. Human challenge studies to accelerate coronavirus vaccine licensure. J Infect Dis. 2020;221(11): 1752-6.

26. Schaefer GO, Tam CC, Savulescu J, Voo TC. COVID-19 vaccine development: time to consider SARS-CoV-2 challenge studies? Vaccine. 2020;38(33):5085-8.

27. Meagher KM, Cummins NW, Bharucha AE, Badley AD, Chlan LL, Wright RS. COVID-19 ethics and research. Mayo Clin Proc. 2020;95(6):1119-23.

28. Verity R, Okell LC, Dorigatti I, Winskill P, Whittaker C, Imai N, et al. Estimates of the severity of coronavirus disease 2019: a model-based analysis. Lancet Infect Dis. 2020;20(6):669-77.

29. Resnik DB. Limits on risks for healthy volunteers in biomedical research. Theor Med Bioeth. 2012;33(2):137-49.

30. Gerlich WH. Medical virology of hepatitis B: how it began and where we are now. Virol J. 2013;10:239.

Publisher's note Springer Nature remains neutral with regard to jurisdictional claims in published maps and institutional affiliations. 